\title{
Model-Based Registration for Motion Compensation during EP Ablation Procedures
}

\author{
Alexander Brost ${ }^{1 \star \star}$, Rui Liao ${ }^{2}$, Joachim Hornegger ${ }^{1}$, and Norbert Strobel ${ }^{3}$ \\ 1 Pattern Recognition Lab, Department of Computer Science, \\ Friedrich-Alexander-University Erlangen-Nuremberg, Erlangen, Germany, \\ 2 Siemens Corporate Research, Imaging and Visualization, Princeton, NJ, USA, \\ 3 Siemens AG, Forchheim, Germany.
}

\begin{abstract}
Radio-frequency catheter ablation (RFCA) has become an accepted treatment option for atrial fibrillation (Afib). RFCA of Afib involves isolation of the pulmonary veins under X-ray guidance. For easier navigation, two-dimensional X-ray imaging may take advantage of overlay images derived from static pre-operative $3-\mathrm{D}$ data set to add anatomical details which, otherwise, would not be visible under X-ray. Unfortunately, respiratory and cardiac motion may impair the utility of static overlay images for catheter navigation. We developed a system for image-based 2-D motion estimation and compensation as a solution to this problem. It is based on 2-D catheter tracking facilitated by modelbased registration of an ellipse-shaped model to fluorosocpic images. A mono-plane or a bi-plane X-ray C-arm system can be used. In the first step of the method, a 2-D model of the catheter device is computed. Respiratory and cardiac motion at the site of ablation is then estimated by tracking the catheter device in fluoroscopic images. The cost function of the registration step is based on the average distance of the model to the segmented circumferential mapping catheter using a distance map. In our experiments, the circumferential catheter was successfully tracked in 688 fluoroscopic images with an average 2-D tracking error of 0.59 $\mathrm{mm} \pm 0.25 \mathrm{~mm}$. Our presented method achieves a tracking rate of 10 frames-per-second.
\end{abstract}

\section{Introduction}

In the United States about two million people are affected by some form of atrial fibrillation (AF), making AF the most common sustained heart arrhythmia and a leading cause of stroke. Radio-frequency catheter ablation (RFCA) has become an accepted option for treating AF in today's electrophysiology (EP) labs, especially, if drug treatment has become ineffective $[1,2]$. RFCA of the pulmonary veins (PVs) usually requires fluoroscopic guidance. Unfortunately, $\mathrm{X}$-ray images cannot distinguish soft tissue well. To address this issue, image integration combining pre-operative 3-D atrial CT data or MR volumes with the fluoroscopic images has been developed, commonly known as fluoroscopic overlay

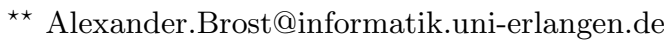


image guidance. The advantage of this strategy is the fused display of the actual, real-time fluoroscopic images together with the highly detailed soft-tissue images from CT or MRI [3-6]. In fact, state-of-the art C-arm systems [7,8], facilitate 3 -D tomographic reconstruction. In other words, the fluoroscopic C-arm device itself can be used to obtain volumetric data sets, e.g., of the heart [9-14]. Since the 3-D data has been acquired on the same device that is used for 2-D X-ray imaging, initial registration of the 3 -D data set to the 2-D fluoroscopic projection can be accomplished. The use of data sets from other modalities, e.g., CT, MR or even 3-D ultra-sound is possible. However, 2-D/3-D registration needs to be performed.

Current fluoroscopic overlay techniques are, however, static, i.e., they do not follow the heart while it beats and moves through the breathing cycle. To achieve a dynamic fused visualization, we need to take this motion into account. Unfortunately, there are few discernible features in typical EP fluoroscopic images. A first approach for 3-D respiratory motion compensation based on 3-D device tracking was proposed by the authors in [15], but this approach required continuous bi-plane fluoroscopy increasing the amount of X-ray dose. In this paper, we describe a method that requires only mono-plane fluoroscopy and therefore works in 2-D. To perform motion estimation, we still track the circumferential mapping catheter, a commonly available EP catheter. Since this device is often used for PV ablations, there is no need for additional instruments or fiducial markers. In addition, the mapping catheter is of unique shape, and it is one of the most prominent structures shown in EP fluoroscopy scenes, representing a good feature for robust tracking. During pulmonary vein isolation, the circumferential mapping catheter is typically fixed at the ostium of the PV that is to be ablated. Hence, by tracking the circumferential mapping catheter, we can obtain a motion estimate right at the ablation site. Once an estimate of the 2-D motion is available, we can, e.g., apply it to the static fluoroscopic overlay image to generate an animated representation of it moving in sync with the tracked device.

\section{Two-Dimensional Model Generation}

The circumferential mapping catheter on the imaging plane is extracted by manual clicking followed by fast marching in the first frame of the fluoroscopy sequence, as explained in [16]. This step provides the points $\mathbf{p}_{i}=\left(u_{i}, v_{i}\right)^{T}$ with $i=1, \ldots, N$ of the catheter device. The 2 -D ellipses are then calculated such that all ellipse points satisfy the linear equation [17]

$$
a u_{i}^{2}+b u_{i} v_{i}+c v_{i}^{2}+d u_{i}+e v_{i}+f=0
$$


with the 2-D coordinates $u$ and $v$. The points $\mathbf{p}_{i}$ of the catheter are combined in a measurement matrix [18]

$$
\mathbf{M}=\left(\begin{array}{cccccc}
u_{1}^{2} & u_{1} v_{1} & v_{1}^{2} & u_{1} & v_{1} & 1 \\
\vdots & \vdots & \vdots & \vdots & \vdots & \vdots \\
u_{i}^{2} & u_{i} v_{i} & v_{i}^{2} & u_{i} & v_{i} & 1 \\
\vdots & \vdots & \vdots & \vdots & \vdots & \vdots \\
u_{N}^{2} & u_{N} v_{N} & v_{N}^{2} & u_{N} & v_{N} & 1
\end{array}\right) .
$$

Then Eq. (1) can be rewritten as

$$
\mathbf{M} \cdot \mathbf{f}=0
$$

with the implicit ellipse parameters $\mathbf{f}=(a, b, c, d, e, f)^{T}$. As the points may not necessarily lie exactly on the ellipse to be fitted, we are looking for the ellipse parameters $\hat{\mathbf{f}}$ that minimize

$$
\hat{\mathbf{f}}=\arg \min _{\mathbf{f}}\|\mathbf{M f}\|_{2}^{2}
$$

subject to

$$
b^{2}-4 a c<0 .
$$

Errors in the points $\mathbf{p}_{i}$ used for the measurement matrix $\mathbf{M}$ lead to different estimated parameters $\hat{\mathbf{f}}$. Since a fast-marching algorithm is used to extract many points along the catheter in the fluoroscopic image, user errors due to inaccurate clicking, have rather little impact. Unfortunately, the constraint, $\|\mathbf{f}\|_{2}=1$, commonly used for ellipse fitting [18], does not necesarily guarantee an elliptic solution. Therefore, the method presented in [17] has been applied. It proposes to use the constraint $b^{2}-4 a c<0$ to assure an elliptical solution $[19,20]$.

Given the implicit ellipse parameters $\hat{\mathbf{f}}=(\hat{a}, \hat{b}, \hat{c}, \hat{d}, \hat{e}, \hat{f})^{T}$, the explicit parameters can be calculated. To this end, we use the following substitution

$$
(\hat{a}, \hat{b}, \hat{c}, \hat{d}, \hat{e}, \hat{f})^{T}=(\tilde{a}, 2 \tilde{b}, \tilde{c}, 2 \tilde{d}, 2 \tilde{e}, \tilde{f})^{T} .
$$

The center of the ellipse $\mathbf{q}_{c}=\left(u_{c}, v_{c}\right)^{T}$ is calculated by [21-23]

$$
\begin{aligned}
& u_{c}=\frac{\tilde{b} \tilde{e}-\tilde{c} \tilde{d}}{\tilde{a} \tilde{c}-\tilde{b}^{2}} \\
& v_{c}=\frac{\tilde{b} \tilde{d}-\tilde{a} \tilde{e}}{\tilde{a} \tilde{c}-\tilde{b}^{2}} .
\end{aligned}
$$

The lengths of the semi-axes, $l_{1}$ and $l_{2}$, are then calculated by

$$
\begin{aligned}
& l_{1}=\sqrt{\frac{\left|2\left(\tilde{a} \tilde{c} \tilde{f}+2 \tilde{b} \tilde{d} \tilde{e}-\tilde{a} \tilde{e}^{2}-\tilde{c} \tilde{d}^{2}-\tilde{f} \tilde{b}^{2}\right)\right|}{\left|\left(\tilde{a} \tilde{c}-\tilde{b}^{2}\right) \cdot\left(\tilde{a}+\tilde{c}+\sqrt{(\tilde{c}-\tilde{a})^{2}+4 \tilde{b}^{2}}\right)\right|}} \\
& l_{2}=\sqrt{\frac{\left|2\left(\tilde{a} \tilde{c} \tilde{f}+2 \tilde{b} \tilde{d} \tilde{e}-\tilde{a} \tilde{e}^{2}-\tilde{c} \tilde{d}^{2}-\tilde{f} \tilde{b}^{2}\right)\right|}{\left|\left(\tilde{a} \tilde{c}-\tilde{b}^{2}\right) \cdot\left(-\tilde{a}-\tilde{c}+\sqrt{(\tilde{c}-\tilde{a})^{2}+4 \tilde{b}^{2}}\right)\right|}} .
\end{aligned}
$$


The rotation within the 2-D image plane is given as

$$
\gamma=\frac{1}{2} \arctan \left(\frac{2 \tilde{b}}{\tilde{c}-\tilde{a}}\right) .
$$

The explicit ellipse parameters are summarized in Figure 1. Discrete sample points $\mathbf{q}_{\theta}$ of the ellipse can then be obtained by [24]

$$
\mathbf{q}_{\theta}=\mathbf{q}_{c}+l_{1} \cdot \cos \theta \cdot \mathbf{R}_{\gamma} \mathbf{e}_{1}+l_{2} \cdot \sin \theta \cdot \mathbf{R}_{\gamma} \mathbf{e}_{2} .
$$

To this end, the parameter $\theta \in[0,2 \pi]$ is used for computing points along the ellipse, i.e., each $\theta$ produces a $\mathbf{q}_{\theta}$. The unit vectors of the coordinate system are called $\mathbf{e}_{1}=(1,0)^{T}$ and $\mathbf{e}_{2}=(0,1)^{T}$. The 2 -D rotation matrix is denoted as

$$
\mathbf{R}_{\gamma}=\left(\begin{array}{cc}
\cos \gamma-\sin \gamma \\
\sin \gamma & \cos \gamma
\end{array}\right)
$$

The model may not always fit perfectly. The difference between model and the actual catheter is called model error. It is obtained by calculating the distance of the model to a gold standard segmentation in the first frame of a sequence. The model error is the difference between the manually outlined catheter and the fitted ellipse. An illustration of this idea is presented in Figure 2.

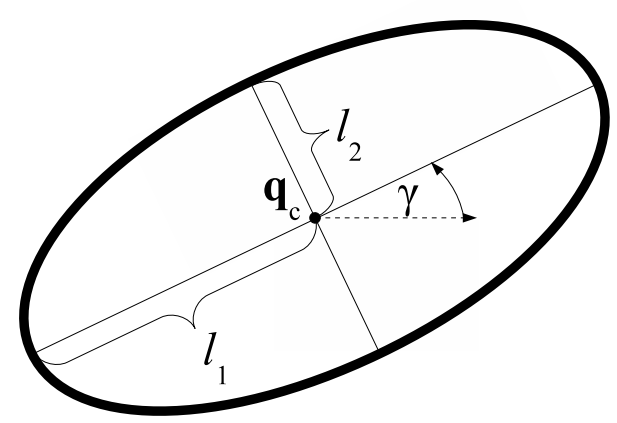

Fig. 1. An ellipse is described by its center, $\mathbf{q}_{c}$, its semi-axes, $l_{1}$ and $l_{2}$, and its rotation in the $2-\mathrm{D}$ plane, $\gamma$.

\section{Catheter Tracking by Registration}

\subsection{Feature Extraction and Distance Map Calculation}

After the catheter model has been generated from the first frame of the fluoroscopic sequence, it is tracked throughout the remainder of the sequence. To 


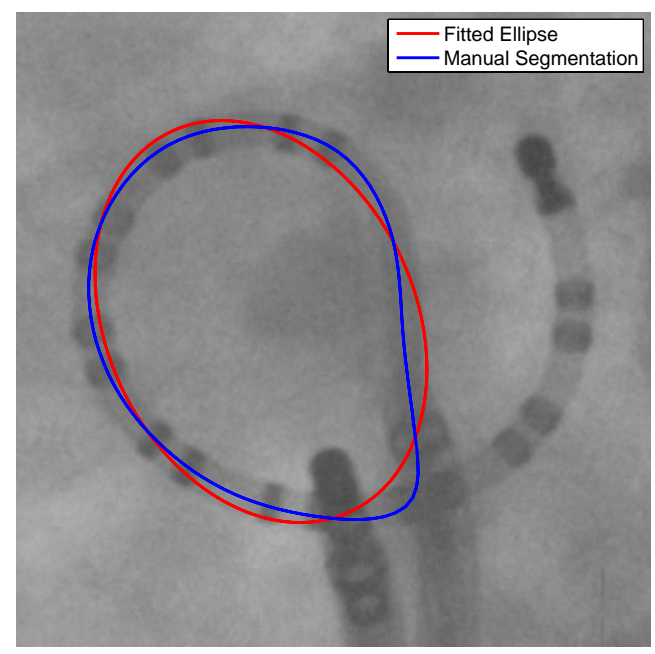

Fig. 2. Illustration of the model error for the first frame of clinical sequence 10 . The manual segmentation is taken as a gold standard rerference. The resulting model error is $0.78 \mathrm{~mm}$ in this case. The model error is the difference between the manually outlined catheter and the fitted ellipse.

speed up the computational efficiency and to minimize the influence of peripheral structures that could interfere with catheter tracking, the region of interest (ROI) for tracking is restricted to $400 \times 400$ pixels (on the $1024 \times 1024$ image) around the center of the tracked mapping catheter in the previous frame. Histogram equalization is further applied on the ROI to enhance the structure of the catheter. Next, a vessel enhancement filter as proposed in [25] is used to improve line-like structures such as the circumferential mapping catheter. The feature image is then binarized using Otsu's method [26] to facilitate segmentation of the mapping catheter. As the model has only a diameter of one pixel, we further apply a skeletonization algorithm as proposed in [27] to thin the segmentation. Finally, a distance map is calculated from the binarized image [28]. A distance map encodes the distance from a point to its closest feature point, that is the nonzero point representing the extracted mapping catheter in our binarized feature image. The distance transform offers an important advantage. It provides a denoised representation of the fluoroscopic image with a pronounced minimum around the shape of the mapping catheter. The distance map is denoted as $\mathbf{I}_{\mathrm{DT}}$, with $\mathbf{I}_{\mathrm{DT}}(\mathbf{p})$ returning the distance of pixel position $\mathbf{p}$ to the segmented catheter. The image processing steps are summarized in Figure 3. 


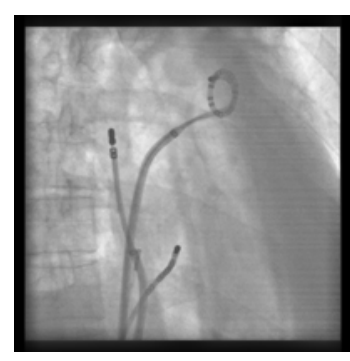

(a) Original Image

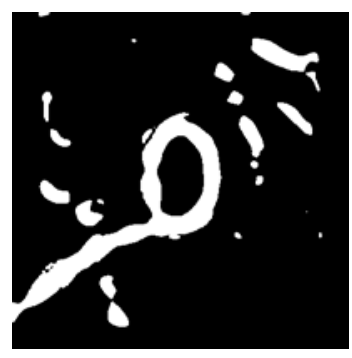

(d) Filtered Image

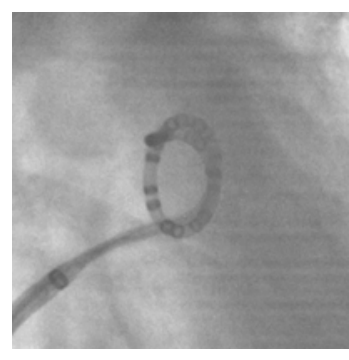

(b) Cropped Image

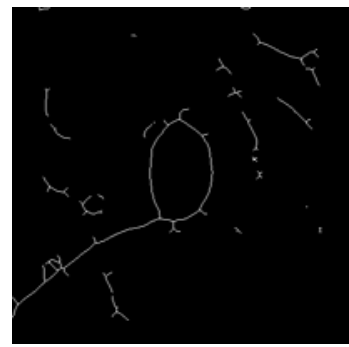

(e) Thinned Image

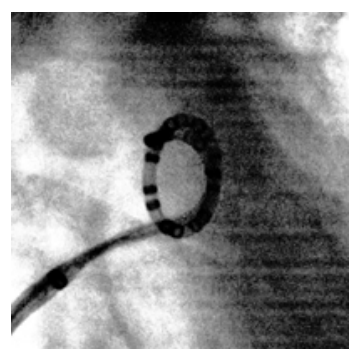

(c) Equalized Image

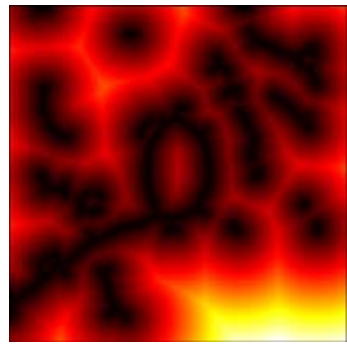

(f) Distance Map

Fig. 3. Image processing steps to enhance the elliptical structure of the catheter. This is needed to obtain a good registration. First, the acquired fluoroscopic image is cropped. The second step involves histogram equalization to enhance semi-transparent parts of the catheter. Afterwards, a vessel enhancement filter is applied to improve the structure of the catheter. It is then binarized using Otsu's method. As the catheter is usually wider than the model, a thinning algorithm is also applied. A distance map is computed next based on the thinned catheter representation. The final distance map provides a smooth image for model-based registration.

\subsection{Model-Based Registration}

Circumferential catheter model tracking is achieved by performing model-based registration. The catheter model is translated by $\mathbf{t}=\left(\Delta_{u}, \Delta_{v}\right)$. The offset in $u$ direction is called $\Delta_{u} \in \mathbb{Z}$, and the offset in $v$-direction is referred to as $\Delta_{v} \in \mathbb{Z}$. The average distance between catheter model and segmentation in the fluoroscopic image is then considered as the cost value. It is calculated by using the distance map introduced above. The optimal translation $\hat{\mathbf{t}}$ is found by optimizing

$$
\hat{\mathbf{t}}=\arg \min _{\mathbf{t}} \sum_{\theta} \mathbf{I}_{\mathrm{DT}}\left(\mathbf{q}_{\theta}+\mathbf{t}\right)
$$

with the distance map $\mathbf{I}_{\mathrm{DT}}$ and $\theta$ as explained in the context of Eq. 12 above. The best parameters are found by nearest neighbor optimization, i.e., the position 
of the local optimum on a large scale is used as starting point for the next optimization on a smaller scale. We start with a $400 \times 400$ ROI and perform optimization with a step size of 16 pixels in each direction. Once we reach a local optimum, we reduce the step size to one pixel in $u$ and $v$ direction on a $16 \times 16$ sub-ROI. Since the shape of mapping catheters may not be exactly elliptical, a simple elliptical model may not always fit perfectly. Thanks to the properties of the distance map, we still end up with a good solution in most cases. A nearest neighbor optimizer is used to iteratively optimize the translational parameters. The estimated 2-D translation $\hat{\mathbf{t}}$ can be directly applied to the 2-D overlay to move it in sync with the tracked device.

\section{Experimental Evaluation and Results}

\begin{tabular}{|r|c|c|}
\hline No. & Mean \pm Std. & Model Error \\
\hline \hline 1 & $0.40 \mathrm{~mm} \pm 0.04 \mathrm{~mm}$ & $0.39 \mathrm{~mm}$ \\
\hline 2 & $0.80 \mathrm{~mm} \pm 0.11 \mathrm{~mm}$ & $0.67 \mathrm{~mm}$ \\
\hline 3 & $0.63 \mathrm{~mm} \pm 0.13 \mathrm{~mm}$ & $0.37 \mathrm{~mm}$ \\
\hline 4 & $0.47 \mathrm{~mm} \pm 0.09 \mathrm{~mm}$ & $0.37 \mathrm{~mm}$ \\
\hline 5 & $0.32 \mathrm{~mm} \pm 0.04 \mathrm{~mm}$ & $0.29 \mathrm{~mm}$ \\
\hline 6 & $0.63 \mathrm{~mm} \pm 0.15 \mathrm{~mm}$ & $0.30 \mathrm{~mm}$ \\
\hline 7 & $0.92 \mathrm{~mm} \pm 0.26 \mathrm{~mm}$ & $0.59 \mathrm{~mm}$ \\
\hline 8 & $0.63 \mathrm{~mm} \pm 0.10 \mathrm{~mm}$ & $0.56 \mathrm{~mm}$ \\
\hline 9 & $0.48 \mathrm{~mm} \pm 0.05 \mathrm{~mm}$ & $0.54 \mathrm{~mm}$ \\
\hline 10 & $1.05 \mathrm{~mm} \pm 0.20 \mathrm{~mm}$ & $0.87 \mathrm{~mm}$ \\
\hline 11 & $0.46 \mathrm{~mm} \pm 0.07 \mathrm{~mm}$ & $0.42 \mathrm{~mm}$ \\
\hline 12 & $0.77 \mathrm{~mm} \pm 0.22 \mathrm{~mm}$ & $0.53 \mathrm{~mm}$ \\
\hline 13 & $0.66 \mathrm{~mm} \pm 0.11 \mathrm{~mm}$ & $0.52 \mathrm{~mm}$ \\
\hline 14 & $0.55 \mathrm{~mm} \pm 0.16 \mathrm{~mm}$ & $0.35 \mathrm{~mm}$ \\
\hline 15 & $0.60 \mathrm{~mm} \pm 0.12 \mathrm{~mm}$ & $0.50 \mathrm{~mm}$ \\
\hline 16 & $0.69 \mathrm{~mm} \pm 0.10 \mathrm{~mm}$ & $0.58 \mathrm{~mm}$ \\
\hline 17 & $0.64 \mathrm{~mm} \pm 0.15 \mathrm{~mm}$ & $0.51 \mathrm{~mm}$ \\
\hline \hline $\boldsymbol{\mu}$ & $\mathbf{0 . 5 9} \mathbf{~ m m ~} \pm \mathbf{0 . 2 5} \mathbf{m m}$ & $\mathbf{0 . 4 9} \mathbf{~ m m}$ \\
\hline
\end{tabular}

Table 1. Average tracking error for the clinical sequences used. The last row shows an average over all the 17 sequences. The total number of frames was 688 .

We evaluated our algorithm by calculating the tracking error for each Xray image over seven different clinical fluoroscopy sequences that were acquired during EP procedures on an AXIOM Artis dBA C-arm system (Siemens AG, Forchheim, Germany). Although our data was acquired on a bi-plane system, our motion estimation approach is not restricted to such a system and will work on a mono-plane device as well. We focus on a typical setup involving a circumferential mapping catheter and one ablation catheter only. The presence 
of other structures should not decrease the accuracy of our method, because we use an unique elliptical structure for registration. To evaluate the tracking error, we calculated the average distance of the forward tracked catheter model to a manually segmented circumferential mapping catheter. The manual catheter segmentation was supervised by a cardiologist, and we consider it our reference result. This distance was averaged over all frames of a particular sequence to arrive at an overall tracking error for each sequence. We tested our method on 17 clinical data sets. The results are summarized in Table 1 . The average of the mean tracking error over all 17 sequences - 688 frames - was $0.59 \mathrm{~mm}$. Figure 5 shows six frames of sequence 13. Our method currently achieves a frame rate of 10 frames-per-second using a (single threaded) CPU implementation. This is sufficiet for clinical applications. In addition to the tracking error, a model error can be calculated. The model error can be obtained from the first image of a sequence as no registration was performed in this particular frame. The model errors ranged between $0.31 \mathrm{~mm}$ and $0.78 \mathrm{~mm}$. A detailed frame-by-frame tracking error for sequence no. 13 is presented in Fig. 4.

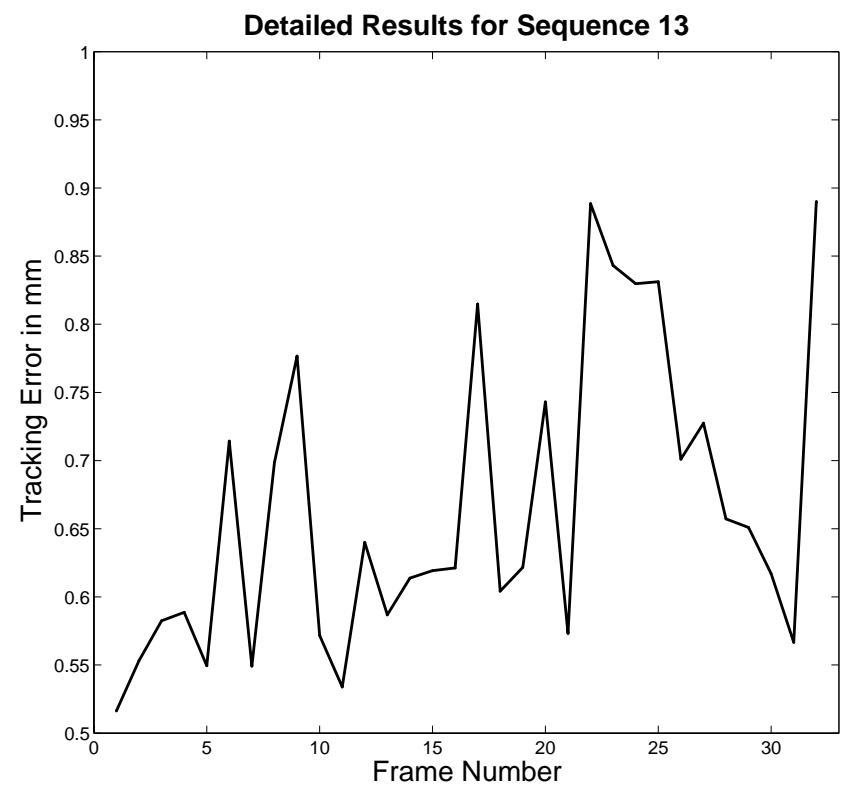

Fig. 4. Frame-by-frame tracking error in $\mathrm{mm}$ for sequence no. 13. The average tracking error is $0.66 \mathrm{~mm} \pm 0.11 \mathrm{~mm}$. The model error for this sequence is $0.52 \mathrm{~mm}$. 


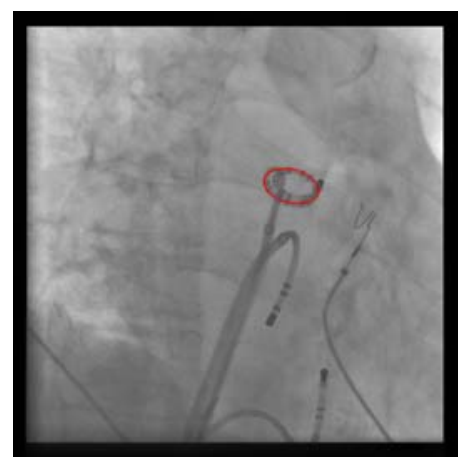

(a) Frame 5

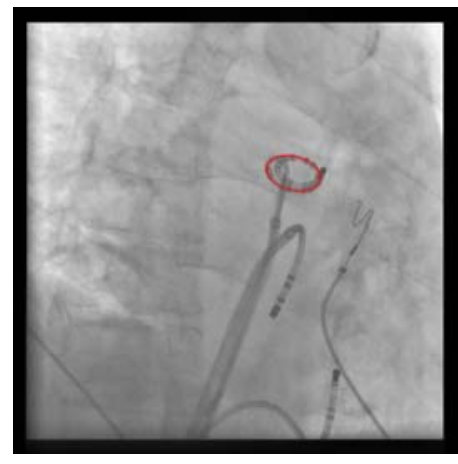

(c) Frame 15

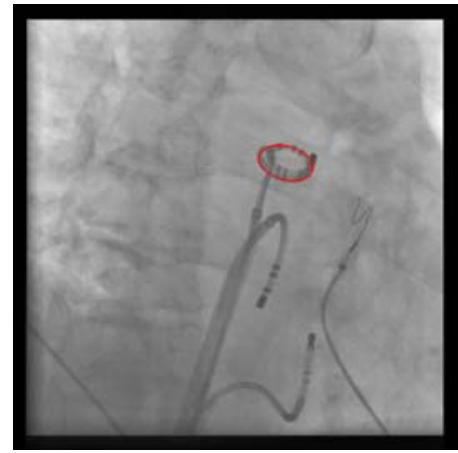

(e) Frame 25

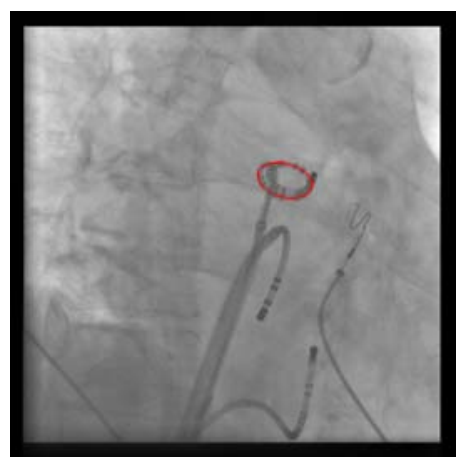

(b) Frame 10

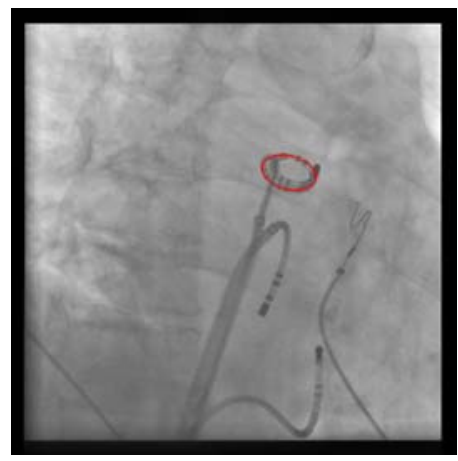

(d) Frame 20

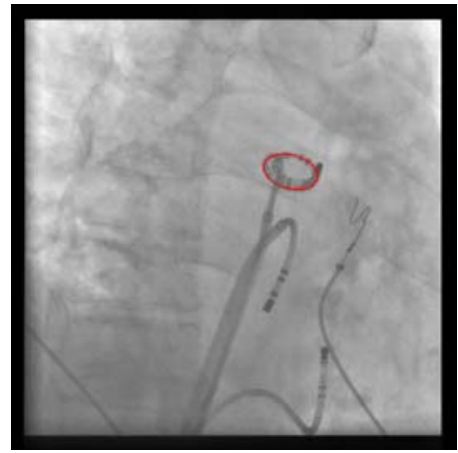

(f) Frame 30

Fig. 5. Six frames of sequence no. 13. The red ellipse shows the tracked circumferential mapping catheter. It aligns well with the outer part of the (spiral) mapping catheter. 


\section{Discussion and Conclusions}

We developed a method for respiratory and cardiac motion estimation for radiofrequency catheter ablation of atrial fibrillation. A C-Arm X-ray system is used to image a circumferential (or spiral) mapping catheter. Catheter tracking is based on a model-based registration framework. We start by estimating a catheter model from the first frame of a fluoroscopic sequence. This model is then tracked throughout the remainder of the sequence using model-based registration. The registration is based on a distance map derived from the fluoroscopic image of the catheter. Our experiments on clinical EP fluoroscopy sequences show that the mean 2-D tracking error is $0.59 \mathrm{~mm}$ including an average model error of $0.49 \mathrm{~mm}$. Considering that breathing motion in typical EP fluoroscopy images is in the range of $15 \mathrm{~mm}$ and for for deep breathing in some patients up to $40 \mathrm{~mm}$, our method has the potential to significantly improve the accuracy of fluoroscopy overlay techniques for EP navigation. The proposed method offers several advantages. First, it is workflow-friendly and does not require fiducial markers or additional contrast agent to be administered. Second, respiratory and cardiac motion is estimated directly at the ablation site. Due to the fact that registration is used, motion estimation and compensation is essentially done in one step. Third, we estimate the motion online and update it constantly from fluoroscopy with a frame rate up to 10 frames-per-second. Furthermore, we do not rely on a predefined motion model from which the real motion may deviate significantly during the procedure. Fourth, there are no restrictions on the 3-D data set that can be used as a fluoro overlay. A 3-D data set could come from MRI, CT or C-arm CT, e.g., syngo DynaCT Cardiac (Siemens AG, Forchheim, Germany). Unlike a previous approach that involved 3-D tracking based on bi-plane imaging [15], the proposed method tracks a device in a 2-D mono-plane fluoroscopic sequence. This method puts fewer restrictions on model generation and registration, since there is no second view to match against. As a consequence, it is possible to obtain a better fit of the model in the 2D fluoroscopic images, i.e., a lower 2-D tracking error. However, this does not necessarily imply better 3-D tracking. Nevertheless, since the motion of the LA can be approximated by a 3-D rigid-body transform [29] and because the LA offers only limited space to move about, we expect that a 2-D motion estimate may offer an acceptable approximation. How well 2-D tracking of a device and a 3-D tracking, however, really relate to each other during EP procedures is subject of further studies.

\section{Acknowledgements}

The authors would like to thank Dr. med. Johannes Rieber for the gold-standard catheter segmentation, Andreas Wimmer for a tool to manually outline catheters easily, and Teri Moore for collecting the clinical data. 


\section{References}

1. Cappato, R., Calkins, H., Chen, S.A., Davies, W., Iesaka, Y., Kalman, J., Kim, Y.H., Klein, G., Packer, D., Skanes, A.: Worldwide Survey on the Methods, Efficacy, and Safety of Catheter Ablation for Human Atrial Fibrillation. Circulation - Journal Of The American Heart Association 111 (2005) 1100-1105

2. Wazni, O.M., Marrouche, N.F., Martin, D.O., Verma, A., Bhargava, M., Saliba, W., Bash, D., Schweikert, R., Brachmann, J., Gunther, J., Gutleben, K., Potenza, E.P.D., Fanelli, R., Raviele, A., Themistoclakis, S., Rossillo, A., Bonso, A., Natale, A.: Radiofrequency ablation vs antiarrhythmic drugs as first-line treatment of symptomatic atrial fibrillation: a randomized trial. JAMA 293(21) (January 21 2005) 2634-2640

3. Zagorchev et. al.: Rapid fusion of $2 \mathrm{D}$ x-ray fluoroscopy with $3 \mathrm{D}$ multislice $\mathrm{CT}$ for image-guided electrophysiology procedures. In: Proceedings of SPIE: Medical Imaging 2007. Volume 6509. (2007) pp. 65092B

4. Sra, J., Narayan, G., Krum, D., Malloy, A., Colley, R., Bhatia, A., Dhala, A., Blanck, Z., Nangia, V., Akhtar, M.: Computed Tomography-Fluoroscopy Image Integration-Guided Catheter Ablation of Atrial Fibrillation. Journal of Cardiovascular Electrophysiology 18(4) (2007) 409-414

5. Ector, J., Buck, S.D., Huybrechts, W., Nuyens, D., Dymarkowski, S., Bogaert, J., Maes, F., Heidüchel, H.: Biplane three-dimensional augmented fluoroscopy as single navigation tool for ablation of atrial fibrillation: Accuracy and clinical value. Heart Rhythm 5(7) (March 25 2008) 957-964

6. Knecht, S., Skali, H., O’Neill, M.D., Wright, M., Matsuo, S., Chaudhry, G.M., Haffajee, C.I., Nault, I., Gijsbers, G.H.M., Sacher, F., Laurent, F., Montaudon, M., Corneloup, O., Hocini, M., Haissaguerre, M., Orlov, M.V., Jais, P.: Computed tomography-fluoroscopy overlay evaluation during catheter ablation of left atrial arrhythmia. Europace 10 (2008) 931-938

7. Hertrich, P.H.: Practical Radiography - Principles and Applications. First edn. Publicis Corporate Publishing (May 2005)

8. Oppelt, A., ed.: Imaging Systems for Medical Diagnostics. Second edn. Publicis Corporate Publishing (November 2005)

9. Orlov, M.V., Hoffmeister, P., Chaudhry, G.M., Almasry, I., Gijsbers, G.H.M., Swack, T., Haffajee, C.I.: Three-dimensional rotational angiography of the left atrium and esophagus: a virtual computed tomography scan in the electrophysiology lab? Heart Rhythm 4(1) (2007) 37-43

10. Al-Ahmad, A., Wigstrm, L., Sandner-Porkristl, D., Wang, P.J., Zei, P.C., Boese, J., Lauritsch, G., Moore, T., Chan, F., Fahrig, R.: Time-resolved three-dimensional imaging of the left atrium and pulmonary veins in the interventional suite: A comparison between multisweep gated rotational three-dimensional reconstructed fluoroscopy and multislice computed tomography. Heart Rhythm 5(4) (2008) 513519

11. Nölker, G., Gutleben, K.J., Marschang, H., Ritscher, G., Asbach, S., Marrouche, N., Brachmann, J., Sinha, A.M.: Three-dimensional left atrial and esophagus reconstruction using cardiac C-arm computed tomography with image integration into fluoroscopic views for ablation of atrial fibrillation: Accuracy of a novel modality in comparison with multislice computed tomography. Heart Rhythm 5(12) (2008) $1651-1657$

12. Strobel, N., Meissner, O., Boese, J., Brunner, T., Heigl, B., Hoheisel, M., Lauritsch, G., Nagel, M., Pfister, M., Rührnschopf, E.P., Scholz, B., Schreiber, B., Spahn, M., 
Zellerhoff, M., Klingenbeck-Regn, K.: Imaging with Flat-Detector C-Arm Systems. In Reiser, M.F., Becker, C.R., Nikolaou, K., Glazer, G., eds.: Multislice CT (Medical Radiology / Diagnostic Imaging). Third edn. Springer Berlin / Heidelberg (January 2009) 33-51

13. Pruemmer et al.: Cardiac C-arm CT: a unified framework for motion estimation and dynamic CT. IEEE Transactions on Medical Imaging 28(11) (November 2009) $1836-1849$

14. Lauritsch et al.: Towards cardiac C-arm computed tomography. IEEE Transactions on Medical Imaging 28(7) (July 2006) 922-34

15. Brost, A., Liao, R., Hornegger, J., Strobel, N.: 3-D Respiratory Motion Compensation during EP Procedures by Image-Based 3-D Lasso Catheter Model Generation and Tracking. In: 12th Conference on Medical Image Computing and ComputerAssisted Intervention (MICCAI). (2009) 394-401

16. Liao et. al.: Location Constraint Based 2D-3D Registration of Fluoroscopic Images of CT Volumes for Image-Guided EP Procedures. In: Proceedings of SPIE: Medical Imaging 2008. Volume 6918. (2008) 69182T

17. Halir, Flusser: Numerically Stable Direct Least Squares Fitting Of Ellipses. In: Proceedings of the 6th Conference in Central Europe on Computer Graphics and Visualization. (1998) 253-257

18. Hartley, Zisserman: Multiple View Geometry in Computer Vision. Second edn. Cambridge University Press (2004)

19. Fitzgibbon, A., Pilu, M., Fisher, R.B.: Direct least square fitting of ellipses. IEEE Transactions On Pattern Analysis And Machine Intelligence 21(5) (May 1999) 476-480

20. Fitzgibbon, A.W., Fisher, R.B.: A Buyer's Guide to Conic Fitting. In: BMVC '95: Proceedings of the 6th British conference on Machine Vision. Volume 2., Surrey, UK, BMVA Press (September 1995) 513-522

21. Bronstein, I.N., Semendjajew, K.A., Musiol, G., Mühlig, H.: Taschenbuch der Mathematik. Fifth edn. Verlag Harri Deutsch, Thun und Frankfurt am Main (2001)

22. Poole, D.: Linear Algebra - A Modern Introduction. First edn. Brooks Cole (2002)

23. D.Zwillinger: CRC Standard Mathematical Tables and Formulae. 31st edn. Chapman \& Hall / CRC (2002)

24. Hearn, D., Baker, M.P.: Computer Graphics with OpenGL. Pearson Prentice Hall (2004)

25. Sato et. al.: 3D Multi-Scale Line Filter for Segmentation and Visualization of Curvilinear Structures in Medical Images. Medical Image Analysis 2(2) (1998) $143-168$

26. Otsu, N.: A Threshold Selection Method from Gray-Level Histograms. IEEE Transactions on Systems, Man, and Cybernetics 9(1) (1979) 62-66

27. Cychosz, J.M.: Efficient Binary Image Thinning using Neighborhood Maps. (1994) 465-473

28. Breu et. al.: Linear time Euclidean distance transform algorithms. IEEE Transactions on Pattern Analysis and Machine Intelligence 17 (1995) 529-533

29. Ector et. al.: Changes in Left Atrial Anatomy Due to Respiration: Impact on Three-Dimensional Image Integration During Atrial Fibrillation Ablation. Journal of Cardiovascular Electrophysiology 19(7) (2008) 828-834 